\title{
Perceived Attributions of Discourse Goals for Using Metaphors and Similes by Iranian EFL Undergraduates
}

\author{
Mahmood Hashemian/Mehdi Iravani (Shahrekord University)
}

\begin{abstract}
The bulk of L2 research on figures of speech boils down to the comprehension domain. By contrast, little work has been conducted on the investigation of the discourse goals for the speaker or the writer's figurative production. This study, going beyond the customary metalinguistic approaches to figurative investigation, aimed at shedding light into a number of different discourse goals as they were identified by Iranian EFL undergraduates in sentences containing figurative language based on 4 independent variables: Figure Type, Tenor Concreteness, Context, and Modality. The participants were given a number of sentences, containing both metaphors and similes, and were asked to choose as many 12 discourse goals as possible which they considered to be the reasons for triggering the utterances. The results indicated that the discourse goal of Compare Similarities was more frequently selected for similes than for metaphors by the participants. The Context variable turned out to have significant interaction with the choice of the discourse goals of Provoke Thought, Get Attention, Clarify, and Contrast Differences, whereas the factor of Modality influenced only Add Emphasis. Also, 3 goals, Add Interest, Clarify, and Show Positive Emotion significantly affected Tenor Concreteness. Comparatively, some marked differences were noticed between Iranian L2 learners and native speakers. L2 teachers are, therefore, advised to more emphasize the correct attributions of discourse goals for metaphors and similes.
\end{abstract}

\section{$1 \quad$ Introduction}

Metaphorical use of language is so widespread in our daily life activities that it is almost impossible to do without this figurative language device. Just read, for example, some few lines of a written discourse or a news item, and discover the metaphorical uses of language in abundance (Eerdmans/Di Candia 2007; Kövecses 2002). In fact, Lakoff/Johnson (1980) go far beyond this and contend that "our ordinary conceptual system is basically metaphorical in nature". They believe that we speak metaphorically because our minds are metaphorically "hardwired".

Given that metaphor is of the essence, it is little wonder that there has been a vast body of research on figurative language (e.g., Cameron/Low 1999; Roberts/Kreuz 1994; Steen 2004). After all, those scholars intrigued by this amazing metaphorical dimension of language use are sure to come up with many questions in their minds. They may desire to know about the ways individuals can perceive and produce cognitively a metaphorical piece of language. Specifically, they may long for the adoption of a systematic approach to examine the steps language users take to perceive and produce a text, oral or written, with instances of metaphor.

Of relevance to the aim of the present study is the question of the producer's intention of the metaphorical message and the perception of that intention by the receiver of the message. As 
Harris/Friel/Mickelson (2006) believe, studies on figurative language have taken two sides: comprehension and production. As for the former, the area of L1 and L2 research abounds with miscellaneous studies on the comprehension of figurative use of language, but regarding the latter, upon examining the relevant literature, one would spot areas of neglect in this research domain (Harris et al. 2006). These two research dimensions of metaphor are not on a par in terms of the amount of research done, and quantity of figurative research is weighted in favor of the comprehension side.

The purpose of this study, as such, was to determine those kinds of discourse goals that language receivers perceive as the reasons for the production of figurative language on the part of language producers. The specific focus is on the receivers' assessment of the intentions behind the two uttered figurative devices, namely metaphors and similes.

\section{Background to the Study}

As mentioned in the Introduction part, research studies in the area of figurative language can be generally divided into comprehension (Chiappe/Kennedy/Chiappe 2003; Jones/Estes 2005/2006; Steen 2004; Thibodeau/Durgin 2008) and production (Harris et al. 2006; Roberts/Kreuz 1994) aspects of language use.

To investigate the probable effects of discourse properties on metaphor recognition, Steen (2004) undertook an empirical study of metaphor recognition in which 18 prospective Dutch students of discourse studies were asked to underline any part of a song, called "Hurricane," they thought was metaphorical. The author divided metaphor properties according to a multidimensional discourse approach that distinguishes between linguistic, conceptual, and communicative functions of metaphorical language. A selection of eight structural metaphor variables for each of these discourse functions was chosen. It turned out that there were five effective variables in the song calculated to be good barometers for metaphor recognition: metaphor complexity, level, position in the utterance, text section, and overall text.

Also, Jones/Estes (2005), drawing on the metaphorical class-inclusion model, embarked on a research program with a view to test the extent to which the subjects judged the topic concept (e.g., TIME) to be a member of a vehicle category (e.g., MONEY). They divided the study into three experiments: Experiment 1, Experiment 2, and Experiment 3. In Experiment 1, the participants were asked to judge the degree of membership for topic concept of the vehicle category either for metaphor prime (e.g., That salesman is a bulldozer) or the corresponding literal prime (e.g., That salesman sold a bulldozer). The options available for the participants were 1 for "nonmember," 2 for "partial member," and 3 for "full member". In Experiment 2, the participants were randomly assigned to either a primed or an unprimed condition. The prime factor consisted of an unprimed condition, in which the participants made category membership judgments in the absence of a sentence, and a primed condition in which the sentence (e.g., A donkey is a horse) was provided prior to each categorization question. Finally, in Experiment 3, the researchers examined the effect of metaphor aptness on class inclusion. Chiappe/Kennedy/Smykowski (2003) define aptness as "the extent to which the statement captures important features of the topic". Unlike Gentner/Wolff (1997), Glucksberg (2003) claims that aptness influences metaphor-induced categorization. Taking all the three experiments together, the authors found that metaphor comprehension involves a process of attributive categorization, whether conventional or novel, highly apt or less apt, and regardless of the relational similarity between topic and vehicle.

As for the production side of metaphorical research, which is really few and far between (Harris et al. 2006), a special mention of two major studies (Harris et al. 2006; Roberts/Kreuz 1994) is in order. 
Roberts/Kreuz (cited in Harris et al. 2006) investigated the perceived goals of speakers or writer's discourse goals on the part of listener or reader in using figurative language. In this study, researchers made use of eight figurative language devices: metaphor, simile, hyperbole, idiom, irony, indirect request, understatement, and rhetorical question. Each participant was taught in one single device and then told to provide three extra examples of that kind of figurative device besides naming the reasons for its use. After the experiment, the judges came up with a taxonomy of 19 discourse goals. Among the perceived goals, for instance, hyperbole was found to be associated with such goals as emphasize and clarify.

In a similar vein, in an attempt to make up for a lack of explicitness and proper attention in addressing the perceived discourse goals of the speaker or writer, Harris et al. (2006) conducted a research study examining the perceived reasons by listener or reader as to why a metaphor or simile is used. The participants were 242 native English-speaking students. Like Jones/Estes (2005), the researchers divided their study into three experiments: Experiment 1, Experiment 2, and Experiment 3. In Experiment 1, they primed their subjects with a list of 16 sentences, containing both metaphors and similes, and their subsequent discourse goals, and told them to check those goals they considered to be relevant to the production of the specific figurative sentence. All in all, of the 12 discourse goals, only Compare Similarities, the most frequently chosen goal overall, differed for metaphors and similes. This goal was more often chosen for similes (64\%) than for metaphors (57\%). In Experiment 2, the task was for the subjects to choose between two alternative forms of the same sentence, one a metaphor and the other a simile. In fact, the researchers were interested in the subjects' preference in the choice of wording in sentences. Overall, similes were perceived over metaphors, with mean preferences of $72 \%$ and $28 \%$, respectively. For Experiment 3, like the one by Jones/Estes (2005), the participants were required to judge the degree of aptness for the comparison stated by metaphor or simile. The calculation of mean aptness for each sentence revealed the mean aptness ratings for metaphor $(M=4.88)$ and simile $(M=5.08)$ sentences did not significantly differ. Generally speaking, the two versions of any given sentence did not differ much. If one was relatively low in aptness, the other usually was, too. Thus, the higher attribution of the discourse goal Compare Similarities in Experiment 1 to similes than to metaphors cannot be attributed to similes being a generally more apt means of comparison.

Despite such a great amount of importance attached to this figurative language use, evident in the enormous amount of research done in the area of L1 and L2, one can find almost no cases, to the best of the present researcher's knowledge, of research studies aimed at any systematic investigation of the discourse goals attributed to a speaker's metaphorical utterance on the part of L2 learners. We can only find such a similar work by Harris et al. (2006), with their subjects being merely native English-speaking students. So, the area of interest of the study was exclusively L1 and the findings exclusively generalizable to an L1 context. In a pedagogical perspective, however, the comprehension of reasons by L2 learners for the utterance of a figurative, let's say, sentence containing a metaphor or simile, whether spoken or written, comes to the fore. Obviously, speaking from past experience, L2 English students often have difficulty understanding metaphorical language. One can easily imagine the deplorably bad situation when students come to the teacher and complain of their uncertainty, during a reading process, about the intended reasons for the writer of a simple prose text, or any other academic text, to use a metaphor or simile. Therefore, it stands to good reason to have an investigation into such perceived reasons for a figurative language use in an L2 classroom context, and to draw on the findings accordingly in our teaching career to improve the students' perceptions of figurative language in general. After all, if metaphorical understanding of language use is so important for L2 learner's success, both in their academic studies and in their communication in L2, it makes sense to try and probe into such a cognitive understanding process. Do all students understand the underlying reasons for the utterance of a metaphor in 
the same way? What is the teacher's role in the improvement of such metaphorical perceptions? Given the state-of-the-art in metaphor research, these are serious questions badly in need of convincing answers. In this perspective, thus, the purpose of the present study was to investigate the discourse goals that Iranian L2 learners of English perceive as the reasons for the production of figurative language on the part of language producers. The specific focus was on the receivers' assessment of the intentions behind the two uttered figurative devices, namely metaphors and similes. It is hoped that the results of the study can shed some light on the process involved in metaphorical language production and comprehension in an L2 context. The study, therefore, sought answers to the following null hypotheses:

- $\mathrm{H}_{01}$ : L2 English students do not attribute any different discourse goals to an interlocutor (in speech or writing) for choosing a metaphor over a simile.

- $\mathrm{H}_{02}$ : The presence or absence of a discourse context does not have any effect on perceived attributions of discourse goals.

- $\mathrm{H}_{03}$ : The particular modality of production (i.e., speech or writing) does not have any effect on perceived attributions about an interlocutor's goals in using a particular metaphor or simile.

- $\mathrm{H}_{04}$ : The degree of concreteness of tenor does not have any effect on perceived attributions of discourse goals.

\section{$3 \quad$ Methodology}

\subsection{Participants}

For this study, the participants were selected from a total population of 80 male and female EFL Translation undergraduates, aged 20-25, from Shahrekord University in their fourth year of study. In order to ensure the homogeneous entry behavior of the participants in terms of proficiency, the Oxford Placement Test (OPT), with reasonable measures of validity and reliability, was used to screen the students. The participants who scored lower than $50 \%$ of the total possible score were excluded from the study. This filtering process left the present researchers with 40 participants.

\subsection{Instruments}

The materials were as the followings: The first material was an Oxford Placement Test (OPT) to gauge the participants' proficiency knowledge and, accordingly, to homogenize them prior to the study. The test consisted of 100 items, all assessing the grammatical knowledge of the participants.

The second materials were two lists of 16 sentences each. Half of the sentences in each list were metaphors and half were similes, with the two differing only in the addition of the word "like" in the simile sentences. The two lists of sentences were one and the same except that any sentence which contained a simile in one list (e.g., That lie is like a boomerang) contained a metaphor in the other list (e.g., That lie is a boomerang). All the sentences were in present tense and had concrete vehicles/predicates. Half of them contained concrete tenors/subjects (e.g., The giraffe is (like) a skyscraper), whereas half contained abstract tenors/subjects (e.g., Creativity is (like) a toaster). There were two variations of these two lists. In one type, the sentences were presented in a list format (e.g., Responsibility is (like) shackles), and in the other type, the sentences were embedded at the end of a brief story (e.g., My brother is not a responsible person. You can never depend on him. Last year, for example, he agreed to be our treasurer, and then he suddenly resigned with nobody to replace him. For him, responsibility is like shackles.). 
The answer-sheets consisted of the numbers $1-16$ and a $16 \times 12$ grid of small squares. Across the top were listed 12 discourse goals: Compare Similarities, Add Emphasis, Show Negative Emotion, Provoke Thought, Add Interest, Get Attention, Clarify, Be Humorous, Be Conventional, Be Eloquent, Show Positive Emotion, and Contrast Differences. The participants put an $\mathrm{X}$ in as many of the 12 squares for a given sentence as they thought to indicate the relevant goals for the speaker or writer using that sentence.

\subsection{Procedure}

The design was a mixed $3 \times 2 \times 2 \times 2$. There were two within-subject and two betweensubject variables: Figure Type (metaphor or simile) and Tenor Concreteness (concrete or abstract), and Context (list format or story) and Modality (oral, written, and both), respectively.

The participants were told:

When people speak or write, they choose their words carefully and for particular reasons and effects. For example, if you hear the sentence "Brains are like complex computers," you might think they chose these words to be humorous, to emphasize a point, to provoke thought about a subject, and/or to compare similarities. Similarly, if you hear the sentence "Love is a flower," you might think that the writer chose these words to be eloquent, to show positive emotion, or to provoke thought about a subject. Also, for each metaphorical sentence, one could assume as few or as many discoursal goals as they could think of. There are no right or wrong answers.

The participants were randomly assigned to three context conditions: (1) Oral Group, (2) Written Group, and (3) Oral and Written Group, each containing one-third of the participants. In the Oral Condition, the participants listened to a list of sentences and, for each sentence, were asked to mark all the possible reasons for the speaker choosing the words in their answer-sheets. In the Oral and Both Conditions, the participants both listened and read the list of sentences and marked the possible reasons. This was achieved with the help of a cassette player. The researcher paused the tape recorder after each sentence and waited until all the participants had responded. Then, he continued and switched to the next sentence.

As for the Written Condition, there came the Context variable, namely a list format or a story. Half of the participants read the isolated sentences and marked the possible discoursal goals they thought as appropriate, whereas the other half read the single paragraphs and marked the discoursal goals for the bold underlined sentence embedded at the end of the brief story. The participants at the Story Condition were reminded that they had to select the discourse goals for the final sentence only.

\section{$4 \quad$ Data Analysis}

\subsection{Descriptive Statistics}

Table 5.1 and Figure 5.1 collectively describe the descriptive statistics, frequency and mean, among other important statistical measures, for the 12 chosen discourse goals, depending on their being whether metaphors or similes: 


\begin{tabular}{|l|r|r|r|r|r|r|}
\hline \multicolumn{1}{|c|}{ Discourse Goals } & \multicolumn{1}{c|}{ Minimum } & Maximum & \multicolumn{1}{c|}{ Sum } & Mean & Std. Deviation \\
\hline Compare Similarities M & 36 & .00 & 16.00 & 186.00 & 5.1667 & 4.64451 \\
\hline Compare Similarities S & 36 & 0 & 16 & 287 & 7.97 & 5.091 \\
\hline Add Emphasis M & 36 & 0 & 14 & 168 & 4.67 & 2.849 \\
\hline Add Emphasis S & 36 & 0 & 9 & 119 & 3.31 & 2.303 \\
\hline Negative Emotion M & 36 & 1 & 9 & 139 & 3.86 & 1.807 \\
\hline Negative Emotion S & 36 & 0 & 8 & 138 & 3.83 & 1.978 \\
\hline Provoke Thought M & 36 & 0 & 9 & 115 & 3.19 & 2.227 \\
\hline Provoke Thought S & 36 & 0 & 9 & 106 & 2.94 & 2.042 \\
\hline Add Interest M & 36 & 0 & 8 & 97 & 2.69 & 1.833 \\
\hline Add Interest S & 36 & 0 & 8 & 106 & 2.94 & 1.926 \\
\hline Get Attention M & 36 & 0 & 12 & 158 & 4.39 & 3.147 \\
\hline Get Attention S & 36 & 0 & 12 & 145 & 4.03 & 2.893 \\
\hline Clarify M & 36 & 0 & 6 & 67 & 1.86 & 1.659 \\
\hline Clarify S & 36 & 0 & 6 & 52 & 1.44 & 1.827 \\
\hline Humorous M & 36 & 0 & 9 & 103 & 2.86 & 2.232 \\
\hline Humorous S & 36 & 0 & 14 & 117 & 3.25 & 2.634 \\
\hline Conventional M & 36 & 0 & 14 & 82 & 2.28 & 2.794 \\
\hline Conventional S & 36 & 0 & 14 & 86 & 2.39 & 2.871 \\
\hline Eloquent M & 36 & 0 & 7 & 67 & 1.86 & 1.743 \\
\hline Eloquent S & 36 & 0 & 7 & 65 & 1.81 & 1.737 \\
\hline Positive Emotion M & 36 & 0 & 10 & 144 & 4.00 & 2.715 \\
\hline Positive Emotion S & 36 & 0 & 164 & 4.56 & 2.951 \\
\hline Contrast Differences M & 36 & 0 & 34 & .94 & 1.413 \\
\hline Contrast Differences S & 34 & 0 & 36 & 1.06 & 1.938 \\
\hline Valid N (listwise) & 34 & & & & & \\
\hline & & & & & & \\
\hline
\end{tabular}

Table 1: Descriptive Statistics for the 12 Discourse Goals 


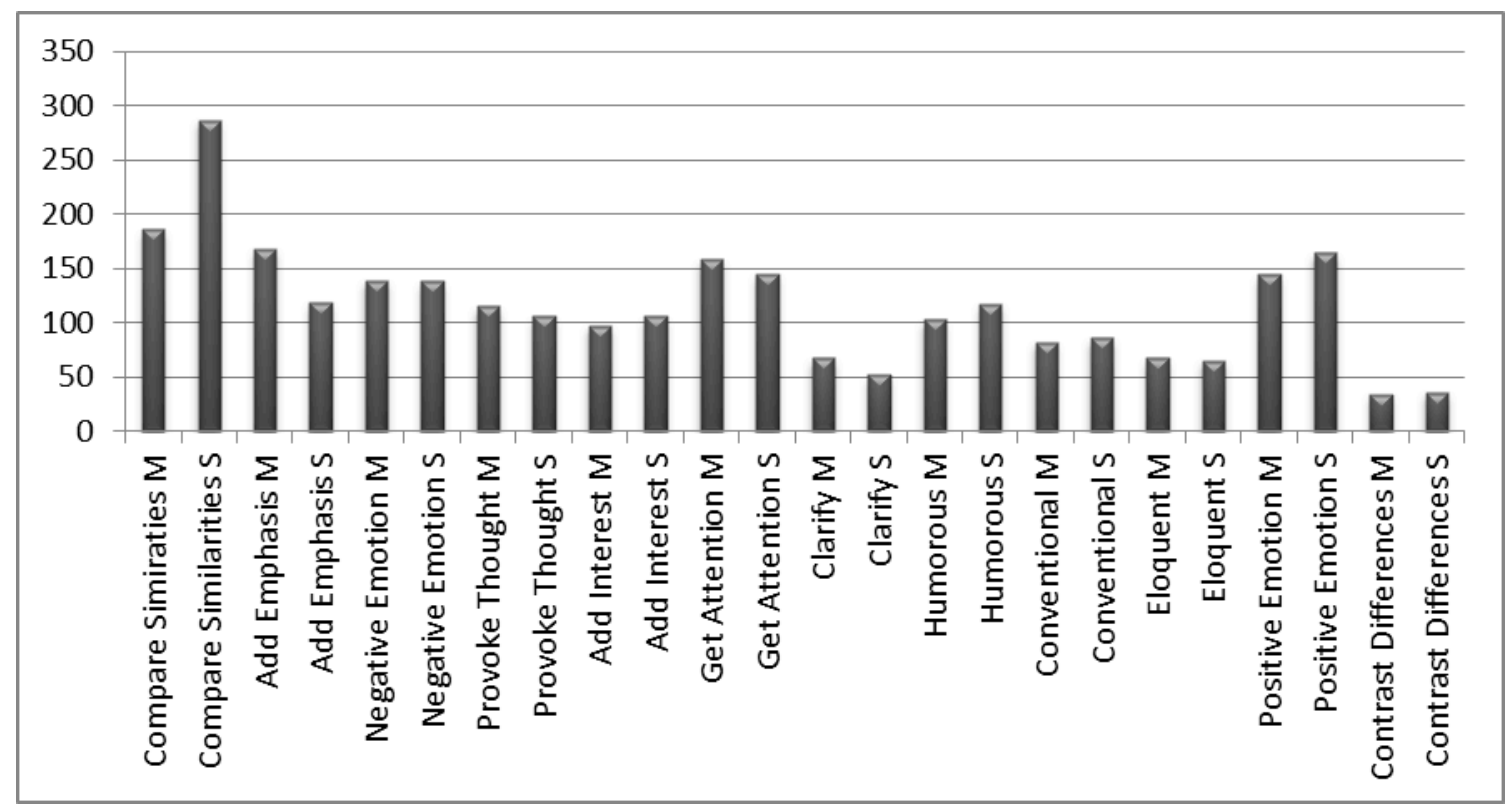

Figure 1: Percent Selecting the 12 Discourse Goals for Both Similes and Metaphors

As depicted above, on balance, out of the 12 discourse goals, the most frequently chosen goal goes with the winner of the battle, namely Compare Similarities in its simile form $(f=287$, $M=7.975, S D=5.091)$. The second to come in the list is naturally the discourse goal of Compare Similarities, but this time in its metaphor form $(f=186, M=5.166, S D=4.644)$. Also, as regards the least frequently chosen goal, Contrast Differences in its metaphor form is the notorious one $(f=34, M=.94, S D=1.413)$.

\subsection{Inferential Statistics}

The data were scored and analyzed for the proportions of each discourse goal marked by the participants. For each discourse goal, the numbers of participants choosing it were analyzed in separate three-way ANOVAs for the between-subjects factors of Modality (Oral, Written, or Both) and Context (Context or List), and the within-subjects factor of Figure (Metaphor or Simile), and also in separate one-way repeated measures ANOVAs for the within-subjects factor of Concreteness (Concrete or Abstract tenor). What follows is the presentation and discussion of the results, with their concomitant tables and figures, for each discourse goal, significant at $p<0.05$.

A main effect for the Figure Type variable was obtained. This was exclusively for one goal, namely Compare Similarities $(F=5.769, d f=1, \alpha=0.05, p=0.019)$. This goal was more frequently chosen for similes $(f=287, M=7.975, S D=5.091)$ than for metaphors $(f=186$, $M=5.166, S D=4.644)$. Since $p$ value is less than $\alpha$, there is a significant difference between the choice of Compare Similarities for metaphors and similes, and, therefore, the first null hypothesis below is rejected:

- L2 English students do not attribute any different discourse goals to an interlocutor (in speech or writing) for choosing a metaphor over a simile.

As for the Context variable, several main effects were found for four discourse goals. This was for four goals, namely Provoke Thought $(F=4.624, d f=1, \alpha=0.05, p=0.035)$, Get Attention $(F=6.190, d f=1, \alpha=0.05, p=0.015)$, Clarify $(F=9.429, d f=1, \alpha=0.05$, $p=0.003)$, and Contrast Differences $(F=5.325, d f=1, \alpha=0.05, p=0.024)$. Since in all these discourse goals $p$ value is less than $\alpha$, the second null hypothesis below is rejected, too: 
- The presence or absence of a discourse context does not have any effect on perceived attributions of discourse goals.

The sentences in Lists were more often than sentences in Context rated as intended to Provoke Thought $(f=108, M=2.94, S D=2.042)$, Get Attention $(f=166, M=4.39, S D=3.147)$, Clarify $(f=74, M=1.86, S D=1.659)$, and Contrast Differences $(f=39, \quad M=1.06$, $S D=1.938)$.

As for the Modality variable, one crucially important effect was found. This was exclusively for the goal Add Emphasis $(F=2.982, d f=2, \alpha=0.05, p=0.044)$. Since, in this discourse goal, $p$ value is less than $\alpha$, the third null hypothesis below is also rejected:

- The particular modality of production (i.e., speech or writing) does not have any effect on perceived attributions about an interlocutor's goals in using a particular metaphor or simile.

The goal was more often chosen in the Both condition $(f=65, M=1.80)$, where the participants had to read and hear the same figurative sentence at the same time. No such effect was found in the Oral or Written conditions.

As for the Concreteness variable, the choice of three goals, suggested by the above tables, turned out to be significant. This was the case for the three goals, namely Add Interest $(F=8.070, d f=1, \alpha=0.05, p=0.008)$, Clarify $(F=3.141, d f=1, \alpha=0.1, p=0.086)$, and Show Positive Emotion $(F=5.567, d f=1, \alpha=0.05, p=0.024)$. Again, since in all these discourse goals, $p$ value is less than $\alpha$, the fourth null hypothesis below is rejected:

- The degree of concreteness of tenor does not have any effect on perceived attributions of discourse goals.

Concrete-tenor sentences were more often than Abstract-tenor sentences regarded as being used to Clarify $(f=68, M=2.00)$, while abstract-tenor sentences were more often than Concrete-tenor sentences seen as Add Interest $(f=113, M=3.32)$, and Show Positive Emotion $(f=169, M=4.97)$.

\section{$5 \quad$ Discussion and Conclusion}

According to the results of the present study, the discourse goal of Compare Similarities was more often chosen for similes than for metaphors by Iranian L2 learners of English. This finding is consistent with the one by Harris et al. (2006), who explain the phenomenon to the effect that since in similes, as distinct from their metaphor counterparts, the functor like clearly signals a very explicit comparison, they definitely stand a higher chance of being chosen by L2 learners as better denoting the idea of similarity between the tenor and the vehicle. This is evidently not the case for metaphors, which are utterly bereft of such a linguistic functor. However, in another study carried out by Roberts/Kreuz (1994), it was the discourse goal of Be Humorous, as against Compare Similarities, that turned out to be chosen more for similes and, hence, distinguished them from their metaphor counterparts. Seemingly, in simile forms, the functor like is implicitly humor-laden (e.g., brains are like complex computers), so that, when read or heard by L2 English learners, it probably acts to trigger off the immediate preference of similes over metaphors. The present study clearly failed to replicate such finding.

Regarding the possible effect of Context variable on the choice of the discourse goals, the findings showed that four discourse goals, out of the 12 ones, namely Provoke Thought, Get Attention, Clarify, and Contrast Differences, were more often chosen in List condition, where single sentences were the only stimulus available to the participants, than in Story condition. This finding is obviously not in agreement with the one by Harris et al. (2006), who found that the two discourse goals of Be Humorous and Compare Similarities were the top choices 
in List condition. Drawing on Kitsch's (1998) framework for levels of representation in memory, the researchers argued that these two goals, being micro-level, are more liable to find their manifestation in text-based level of representation, here, sentence, than in situation model, one-paragraph stories. Back to the present research, one could say that the sentences in the List, in all likelihood, were perceived by the Iranian L2 learners of English to be more intended to Provoke Thought, Get Attention, and Contrast Differences because the sentences were the only stimuli present, and there was no other opportunity for them to proceed to a higher level and to see the overall picture via the situation model representation. They only had the text-based representation level of representation at their disposal. This seems sound, but, what about the choice of the discourse goal of Clarify? Were it in compliance with Kintsch's (1998) framework, it would have been chosen more in Story context than in List condition. Definitely, this does not hold true for Clarify!

Apropos of the possible effect of Modality variable on the choice of the discourse goals, it was found that the Add Emphasis goal was more often chosen by the participants in the Both condition, where the participants read and heard the same figurative sentence at the same time. This replicates exactly Harris et al. (2006), who also found a significant effect of modality on the Add Emphasis goal. One might argue that Add Emphasis is more vividly accentuated and signaled when the discourse is read and heard at the same time. Psycholinguistically speaking, the discourse, in Both condition, unquestionably will become richer in terms of supra-segmental features, namely stress and intonation, hence more drawing the participants' attention and leading their cognition to the inference of emphasis and, subsequently, the choice of Add Emphasis as the best candidate. Relevantly, one intriguing finding by Harris et al. (2006) was the significant interaction between the two variables of Modality and Context, so that the Add Emphasis choice was confined to the List condition. Their claim is that the Story condition is rich enough in terms of context that it is able to do without prosodic cues. In the present study, however, no such interaction was found between any of the four variables.

And finally, in regard to the possible effect of Concreteness factor on the choice of the 12 discourse goals, the discourse goal Clarify, one the one hand, and the discourse goals Add Interest and Show Positive Emotion, on the other hand, turned out to be more often chosen in Concrete-tenor sentences and Abstract-tenor sentences, respectively. As a possible cognitive explanation, one could rationalize this significant effect and claim that Concrete-tenor sentences will put much less mental demand on Iranian L2 learners' engaged mind regarding the juxtaposition of the tenor and the vehicle, both being concrete, and, hence, more readily will direct them to formulate the intention behind the utterance of the sentence as that of clarification. Comparatively, in this case, Abstract-tenor sentences lag behind. These sentences, as put by Harris et al. (2006), due to the inherent differences existing between the abstract tenor and the concrete vehicle, are less probable to be perceived by L2 learners of English as conveying the idea of clarification and simplicity. However, oddly enough, in the present study, the other two discourse goals of Add Interest and Show Positive Emotion which tend to be more chosen in sentences with both concrete tenors and vehicles, hence allowing more cognitive resources available, were chosen more often in the Abstract-tenor sentences. Certainly, this is not the case, at least, for the native speakers of English. To these speakers, as showed by Harris and Mosier (1999), it is mainly the discourse goals of Contrast Differences, Add Emphasis, Provoke Thought, and Show Negative Emotion that more frequently characterize abstracttenor sentences due to the obviously inconsistent natures of the tenors and the vehicles in these sentences. This is a big question! Were one to give a possible explanation, it would seem that Iranian L2 learners just take this added difficulty in the process of abstract, as against concrete, figurative sentences for granted and turn to the less demanding resources to retrieve, such as features of Interest and Positive Emotion. 
To wrap up the discussion, on balance, out of the 12 discourse goals in this study, Compare Similarities was the most frequently chosen goal. All the four independent variables, Figure Type, Tenor Concreteness, Context, and Modality, proved to have affected the choice of the discourse goals. No significant interaction was found between these four variables regarding the choice of discourse goals.

What this research study has brought home vividly is the importance of the interpretation of a piece of figurative discourse, here being metaphor and simile. Obviously, different sociocultural values give rise to different metaphorical interpretations. In this research study, some marked differences were noticed regarding the attributions of discourse goals for similes and metaphors made by Iranian L2 learners and those made by native English speakers, most notably the participants studied by Harris et al. (2006). Definitely, both linguistic and sociocultural differences existing between these two linguistic communities leave some margin for error on the part of the nonnative party, namely Iranian L2 learners. Therefore, it is incumbent upon a teacher to have his or her eyes wide open to this fact and to encourage students to have a unified interpretative version of such figurative devices.

The findings of this study might have implications for L2 pedagogy as well. First, L2 teachers can prepare a detailed profile of various reasons, whether correct or incorrect, different students attribute to different figurative utterances. Based on the original intentions behind these utterances by native speakers, or even L2 teachers' own judgments, L2 teachers can detect their students' areas of weakness and strength in metaphorical understanding and can act accordingly. Obviously, the way different students perceive the same figurative utterance is not the same. Different students with different social and cultural backgrounds certainly transfer their values to the task of L2 learning. Understanding metaphor is one potential area for the manifestation of such sociocultural values. Second, based on a list of most common perceived reasons in one column and their corresponding metaphorical utterances in the other, materials developers can have their own fair share from the implications of this study. They can develop text materials with special emphasis on those aspects of metaphorical understanding which most students find cumbersome to handle. In order to improve L2 learners' perception of reasons for metaphorical sentences, these experts can devise, for example, multiple-choice format exercises which will assess L2 learners' awareness of metaphorical use of language in a piece of context or even a sentence provided in the stem.

\section{References}

Cameron, Lynne/Low, Graham (eds.) (1999): Researching and applying metaphor. Cambridge.

Chiappe, Dan L./Kennedy, John M./Chiappe, Penny (2003): "Aptness is more important than comprehensibility in preference for metaphors and similes". Poetics 31: 51-68.

Chiappe, Dan L./Kennedy, John M./Smykowski, Tim (2003): "Reversibility, aptness, and the conventionality of metaphors and similes". Metaphor and Symbol 8: 85-105.

Eerdmans, Susan L./Di Candia, Michaela (2007): "Watching paint dry: The sequentiality of idiomatic expressions in NS-NS and NS-NNS talk-in-interaction". Discourse Studies 9/5: 579-595.

Gentner, Deidre/Wolff, Philip (1997): "Alignment in the processing of metaphor". Journal of Memory and Language 37: 331-355.

Glucksberg, Sam (2003): "The psycholinguistics of metaphor". Trends in Cognitive Science 7: 92-96.

Harris, Richard Jackson/Friel, Brian M./Mickelson, Nolan Rett (2006): "Attribution of discourse goals for using concrete- and abstract-tenor metaphors and similes with or without discourse context". Journal of Pragmatics 38: 863-879. 
Harris, Richard Jackson/Mosier, Noah J. (1999): "Memory for metaphors and similes in discourse". Discourse Processes 28/3: 257-270.

Jones, Lara L./Estes, Zachary (2005): "Metaphor comprehension as attributive categorization". Journal of Memory and Language 53: 110-124.

Jones, Lara L./Estes, Zachary (2006): "Roosters, robins, and alarm clocks: Aptness and conventionality in metaphor comprehension". Journal of Memory and Language 55: 18-32.

Kintsch, Walter (1998): Comprehension: A Paradigm for Cognition. Cambridge.

Kövecses, Zoltán (2002): Metaphor: A practical introduction. Oxford University Press.

Lakoff, George/Johnson, Mark (1980): Metaphors we live by. Chicago.

Roberts, Richard M./Kreuz, Roger J. (1994): "Why do people use figurative language"? Psychological Science 5: 159-163.

Steen, Gerard (2004): "Can discourse properties of metaphor affect metaphor recognition"? Journal of Pragmatics 36: 1295-1313.

Thibodeau, Paul/Durgin, Frank H. (2008): "Productive figurative communication: Conventional metaphors facilitate the comprehension of related novel metaphors". Journal of Memory and Language 58: 521-540. 